\title{
THE SOUTHERN OBJECTIVE PRISM SURVEY
}

\author{
J. STOCK \\ Instituto Venezolano de Astronomia, Mérida, Venezuela \\ and \\ H. WROBLEWSKI \\ Universidad de Chile, Santiago, Chile
}

\begin{abstract}
Five thousand sq deg of the Southern sky of intermediate to high galactic latitude have been surveyed with deep objective prism plates, taken with a UV-transparent prism. The plates reach on the average the 13th photographic magnitude and have been searched for the following types of objects: (1) high luminosity stars, (2) planetary nebulae, (3) metal weak late-type giants, (4) Me stars, (5) carbon stars, (6) early-type subdwarfs, (7) peculiar objects. Catalogues containing a total of over 3200 objects of these types have been compiled and will soon be published in Publ. Dpto. Astron. Univ. de Chile.

Of particular interest are the metal-weak late-type giants, which are believed to be members of the galactic halo population. Slit spectra were obtained for a number of these stars and radial velocities derived. In each case the object was found to be a high velocity star. In view of the large number of such objects found in the survey, a method of determining relative radial velocities from pairs of objective prism plates was developed. The method, which is described in detail in the following paper, was tested successfully on several plate pairs, confirming the high velocities for the metal weak stars and showing that the method could be used for an exhaustive search for high velocity stars.
\end{abstract}

